\title{
Rolando a Bosta pela Mata Atlântica: Utilização de Infográfico Interativo para o Ensino da Diversidade de Besouros Escarabeíneos
}

\author{
Izabella Neves de Oliveira1, Josival Francisco Araújo1, Fabiana do Carmo \\ Santana2, Rita de Cássia de Moura1, Filipe Martins Aléssio1.
}

1Instituto de Ciências Biológicas, Universidade de Pernambuco, Campus Santo Amaro, Recife, Pernambuco, Brasil.

2Espaço Ciência, Avenida Agamenon Magalhães, Memorial Arcoverde, Complexo de Salgadinho, Olinda, Pernambuco, Brasil.

\footnotetext{
bellaoliveira.neves@gmail.com, araujojosi.f@gmail.com, santanafcr@gmail.com, ritamoura.upe@gmail.com, filipe.martins@upe.br
}

\begin{abstract}
Infographics are a vehicle for transmitting data and information that once joined with visual representation make the process of content assimilation simpler. In view of the communicative power of images in the digital era, it was developed an interactive infographic with information about the Dung beetles present in the Atlantic Forest from the Brazilian Northeastern. With emphasis on its eating habits and ecosystem services, it has a focus the main threats to the biome, and consequently to the beetles. Graphic and informative tools such as infographics can be used in museum's exhibitions, in classrooms, or on websites as a way to stimulate zoologyrelated learning.
\end{abstract}

Resumo. Infográficos são um veículo para transmissão de dados e informações que ao unir-se a representação visual tornam o processo de assimilação do conteúdo mais simples. Tendo em vista o poder comunicativo das imagens em plena era digital, objetivou-se desenvolver um infográfico interativo com informações sobre os besouros rola-bostas presentes na Mata Atlântica Nordestina. Havendo destaque aos seus hábitos alimentares e serviços ecossistêmicos desempenhados, tem em foco as principais ameaças ao bioma, e consequentemente aos besouros. Ferramentas gráficas e informativas como infográficos podem ser utilizados em exposições, em salas de aula ou em sites como forma de estimular o aprendizado relacionado a zoologia.

\section{Introdução}

Infográficos podem ser definidos como representações visuais de informações acompanhadas de texto. De acordo com Niebaum et al. (2015), infográficos são planejados e desenvolvidos, entre outras coisas, para expor e esclarecer informações complexas baseadas em evidências ou resultados de pesquisas científicas e devem alcançar públicos-alvo em locais de fácil acesso. Em educação, experimentos mostram que estudantes que consultam infográficos guardam a informação por mais tempo do que aqueles que consultam apenas texto [Lyra et al. 2016]. A capacidade de interpretar e compreender infográficos são uma competência que deve ser desenvolvida por estudantes na era digital, que navegam na web e devem avaliar informações gráficas e 
mensagens multimídia [Martix and Hodson 2014], encontrando e criando sentidos e conhecimentos através de imagens e mídias visuais [Hattwig et al. 2013]. À medida que as imagens se tornam a forma predominante de comunicação em uma ampla gama de formatos, a imagem e a composição visual têm o poder de moldar nossa compreensão e interpretação de nosso mundo além do literal [Lundy et al. 2015], notadamente em Zoologia, na qual fotos e imagens de animais silvestres despertam curiosidade e fascínio de um público vasto e diversificado.

Os besouros rola-bostas pertencem a subfamília Scarabaeinae (Coleoptera: Scarabaeidae) e são conhecidos popularmente pelo comportamento de rolarem bolas de fezes para nidificação e alimentação [Halffter and Matthews, 1966]. Outros hábitos alimentares além da coprofagia foram registrados em Florestas Tropicais, como a necrofagia, mirmecofilia, saprofagia, carpofagia e a predação [Louzada 2008; Vaz-deMello et al. 1998]. Essa variedade de hábitos permite que os escarabeíneos sejam diversos e ocupem vários nichos nas Florestas Tropicais, que junto com as savanas as vegetações detêm a maior diversidade desse grupo [Hanski and Cambefort, 1991].

A subfamília Scarabaeinae possui aproximadamente 6200 espécies válidas agrupadas em 267 gêneros [Tarasov and Génier, 2015], das quais aproximadamente 726 espécies e 63 gêneros ocorrem no Brasil [Vaz-de-Mello 2019]. São considerados parâmetros para estudo da biodiversidade e utilizados para analisar, inventariar e monitorar a biodiversidade em florestas tropicais [Halffter and Favila 1993]. Muitas espécies de rola-bostas podem ser consideradas indicadoras ambientais, por serem sensíveis a perturbações antrópicas e a destruição do seu habitat [Favila and Halffter 1997]. Devido aos seus hábitos alimentares, esses besouros desempenham importantes serviços ecossistêmicos como a reciclagem de matéria orgânica, incorporação de nutrientes no solo, aeração do solo, dispersão secundária de sementes, controle biológico de moscas hematófagas e detritívoras [Louzada 2008].

O objetivo do presente trabalho é relatar o processo de elaboração de um infográfico sobre os besouros rola-bostas existentes no bioma Mata Atlântica Pernambucana e a sua posterior inclusão no Portal de Zoologia de Pernambuco (www.portal.zoo.bio.br), um site colaborativo de compartilhamento de fotos de animais silvestres.

\section{Material e Métodos}

O infográfico foi produzido no laboratório de informática do Espaço Ciência, Olinda, Pernambuco. A arte do infográfico foi produzida no programa Paint Tool e a editoração do texto no software Photoshop Cs6. Foram utilizados elementos da fauna e da flora local. As espécies de mamíferos foram selecionadas com registros para remanescentes de Floresta Atlântica da Região Metropolitana do Recife. Foram utilizadas imagens de remanescentes da Região Metropolitana do Recife. Os besouros rola-bostas foram selecionados de trabalhos de levantamento de escarabeíneos em Pernambuco [Costa et al. 2013; Araújo et al. 2018] e por exemplares depositados na Coleção Científica de Scarabaeinae do Laboratório de Biodiversidade e Genética de Insetos da Universidade de Pernambuco. Esses besouros foram coletados em Floresta Atlântica utilizando armadilhas de queda do tipo Pitfall que consiste em uma garrafa PET com um porta-isca, iscadas com fezes, carniças ou diplópodes injuriados.

Para incluir o infográfico sobre os besouros rola-bosta no Portal de Zoologia, foi criada uma página desenvolvida exclusivamente para receber a imagem e que a mesma, uma vez aberta, ocupasse todo o espaço da tela para que os detalhes do gráfico sejam observados pelos visitantes do site. O Portal de Zoologia utiliza o sistema modular de publicação SPIP, versão 3.1.10. O sistema pode acolher diferentes tipos de esqueletos 
de páginas web e funciona através da instalação e funcionamento de extensões. Para criar as ligações com registros realizados no Portal, foi utilizada a extensão "Imagem Clicável" 3.0.4 (versão de teste). Esta extensão permite, através da utilização em conjunto da ferramenta "Mapa de imagens" de filtros web do programa Gimp 2.8.22, criar áreas clicáveis específicas em imagens. Tais áreas possuem dimensões totalmente parametrizáveis e podem conter URLs ativas que encaminham os visitantes para outras páginas da Internet.

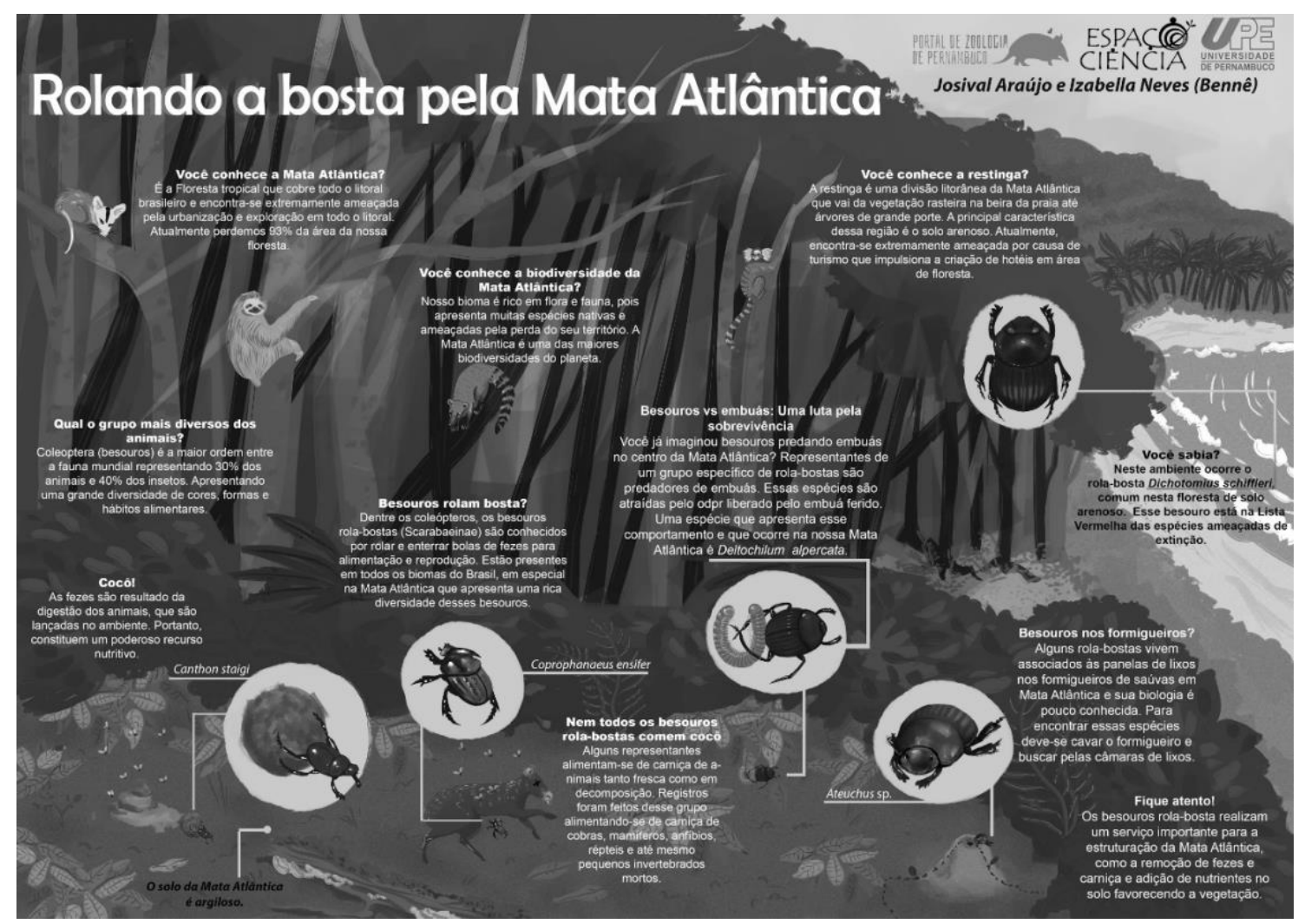

Figura 1. Infográfico "Rolando a Bosta pela Mata Atlântica".

\section{Resultados e Discussão}

Os elementos incorporados no infográfico intitulado "Rolando a bosta pela Mata Atlântica", são o cenário de Floresta Ombrófila Densa e Restinga que faz a transição entre a Mata Atlântica e o litoral e possui fitofisionomias diversas com florestas lenhosas com solo arenoso até uma vegetação rasteiras em praia arenosa. A floresta apresentou copa fechada, no solo foi retratado um elemento comum nas florestas tropicais, a presença de serapilheira e um sub-bosque com uma vegetação rasteira e troncos de árvores caídas com presença de decompositores.

Os elementos da fauna foram divididos em dois grupos: mamíferos e besouros rola-bostas. Os mamíferos retratados foram o timbu, também conhecido como gambáde-orelha-branca, Didelphis albiventris (Lund, 1840), porque essa espécie é comum em áreas urbanas e bastante conhecido pelo público. Outro animal comum em área urbana e em área florestada é o sagui nordestino Callithrix jacchus (Linnaeus, 1758). A preguiça Bradypus variegatus Schinz, 1825, o quati, Nasua nasua (Linnaeus, 1766) e a cutia, Dasyprocta leporina (Linnaeus, 1758) foram representadas. A cutia foi representada morta servindo de recurso para alimentação de besouros necrófagos. Toda essa fauna contribui com recursos, como fezes e carniça, para a comunidade de rola-bostas de área 
florestada. Com o desmatamento e a defaunação, o número de indivíduos mamíferos de grande e médio porte está decrescendo e isso reflete diretamente na estrutura da comunidade de rola-bostas e de modo geral em toda a teia alimentar [Bogoni et al. 2016].

Os hábitos alimentares dos besouros rola-bostas foram relacionandos com o seu recurso, sendo eles coprofagia, necrofagia, predação e mirmecofilia. Na coprofagia os besouros utilizam fezes para alimentação e nidificação e essa guilda funcional desempenha serviços ecossistêmicos como realocação de matéria orgânica, aeração do solo, incorporação de nutrientes no solo e dispersão secundária de sementes dentro das áreas florestadas [Louzada 2008]. A espécie Coprophaneus ensifer (Germar, 1824) é necrófaga e alimenta-se de carniça de animais mortos. No infográfico essa espécie foi representada alimentando-se de uma cutia morta. A coprofagia e a necrofagia são os hábitos alimentares mais comuns dentre os escarabeíneos. No entanto, alguns grupos de rola-bostas ocuparam outros nichos menos competitivos para sobrevivência. Entre eles temos a predação de diplópodes e a mirmecofilia. O gênero Deltochilum (Aganhyboma), possui espécies predadoras de diplópodes que teve seu primeiro registro em Pernambuco em 2018 na Reserva de Floresta Urbana Mata de Jaguarana em Paulista, Pernambuco [Araújo et al. 2018]. A espécie de predador de diplópodes foi representada no infográfico com seu comportamento clássico de arrastar os diplópodes depois de mortos utilizando as pernas posteriores. O hábito alimentar da mirmecofilia ainda não foi registrado no Nordeste brasileiro e consiste em besouros rola-bostas associados a lixeiros de formigas do gênero Atta ou Acromyrmex [Vaz-de-Mello et al. 1998]. A restinga foi representada no infográfico destacando as principais características da vegetação e do solo, assim como da presença de uma espécie endêmica ameaçada desse ambiente, Dichotomius schiffleri (Vaz-de-Mello, Louzada \& Gavino, 2001).

Toda a informação contida no infográfico será exposta permanentemente na Trilha Ecológica do Espaço Ciência, o maior museu de ciência a céu aberto do Brasil. Esse conteúdo também pode ser utilizado em sala de aula, museus, exposições, entre outros. Os infográficos são bastante utilizados em ensino a distância, como um recurso facilitador de ensino-aprendizagem em sala de aula e possui enorme potencial de aplicação no ensino médio e superior [Braga 2009; Taguchi and Ackerman 2014; Cortes et al. 2015]. A infografia também tem um potencial para ser utilizadas na educação inclusiva de pessoas com deficiência auditiva [Lapolli et al. 2014]. Utilizado conjuntamente com o Portal de Zoologia, o infográfico pode se tornar ainda mais interativo, pois estudantes e visitantes em geral poderão contribuir com novos registros das espécies, através notadamente de atividades práticas realizadas em saídas de campo.

O infográfico "Rolando a bosta pela Mata Atlântica" apresenta, em termos de aplicação, duas dimensões didáticas. A primeira dimensão diz respeito à sua utilização prática e direta em projetos pedagógicos em aulas de ciência. A segunda dimensão diz respeito ao processo de desenvolvimento do infográfico. Em relação à primeira dimensão, o uso de novas tecnologias e suas ferramentas, como infográficos, vem aprimorando a aprendizagem e a prática do ensino, fazendo com que os professores utilizem essas novas ferramentas para prepararem os alunos para compreensão e interpretação do conteúdo nos mais diferentes níveis escolares, pois imagens, mesmo sem textos, são uma das formas mais importantes para a apresentação de informações [Johri and Misra 2017; Kibar and Akkoyunlu 2014; Yildirim 2016]. Além disso, evidências sugerem que estudantes preferem infográficos em mídias digitais por apresentarem estruturas interativas [Yidirim 2016]. Desta forma, esse infográfico pode permitir que professores abordem diversos conteúdos específicos da zoologia e ecologia 
em sala de aula, como a biodiversidade da Mata Atlântica, serviços ecossistêmicos, relações interespecíficas, teia alimentar, nicho ecológico, habitat, ciclagem de nutrientes, conservação e nomenclatura zoológica (noções de taxonomia e classificação). Espera-se que o conhecimento apresentado possa garantir uma informação prática, atual e objetiva e que, conectada ao desenho, permita que o conhecimento seja adquirido de forma rápida, clara e duradoura.

Em relação a segunda dimensão didática, a criação de infográficos permite que alunos, notadamente de ensino médio e cursos de graduação, desenvolvam competências visuais e competências de informação, frequentemente de forma concomitante. Competências visuais podem ser consideradas como um "conjunto de habilidades que permitem que um indivíduo possa efetivamente encontrar, interpretar, avaliar, utilizar e criar imagens e mídias visuais". Através dos meios de comunicação atuais, os alunos precisam aprender a ser consumidores e criadores críticos de mídias visuais em geral e como parte de redes de mídias sociais, que muitas vezes se cruzam e se sobrepõem às redes acadêmicas [Hattwig et al 2013]. No contexto de rápidas mudanças tecnológicas e proliferação de fontes de informação, as competências em informação são essenciais para a formação acadêmica. Competências em informação podem ser definidas como um conjunto de habilidades que permitem que o indivíduo reconheça quando a informação é necessária e tenha a capacidade de localizar, avaliar e usar eficazmente a informação. Desta forma, acredita-se que o processo de criação de infográficos digitais é um exercício didático poderoso para que alunos possam desenvolver habilidades pouco exploradas no ensino tradicional focado no professor.

\section{Conclusões}

Esse trabalho apontou a importância da utilização de infográficos como uma poderosa ferramenta para o ensino e divulgação de ciências. O infográfico "Rolando a bosta pela Mata Atlântica" contribuirá para o ensino e divulgação da diversidade e dos serviços ecossistêmicos oferecidos pelos besouros rola-bostas na Floresta Atlântica. Assim, constata-se a importância dos professores utilizarem infográficos em sala de aula, museus e espaços de divulgação de ciência pelo enorme potencial educativo desta ferramenta pouco explorada pela educação. A inclusão de infográficos em sites Internet pode contribuir ainda mais para que estudantes e o público em geral sejam estimulados com conteúdos interativos e que o conhecimento seja construído coletivamente. Como trabalhos futuros, novas edições do infográfico devem ser produzidas em atualização com a literatura e novos registros no Nordeste. Adaptações para diversos públicos devem ser realizadas e jogos para sala de aula serão planejados utilizando esse infográfico.

\section{Referências}

Araújo, J.F., Silva, F.A.B. and Moura, R.C. (2018). Primeiro registro de Deltochilum do subgênero Aganhyboma (COLEOPTERA: SCARABAEIDAE: SCARABAEINAE) em Pernambuco, Brasil. I Congresso Nordestino de Animais Silvestres, UFRPE, Recife.

Braga, C.S. (2009) O Infográfico na Educação a Distância: uma contribuição para a aprendizagem. In: $15^{\circ}$ Congresso Internacional ABED de Educação a Distância. p. 27-30.

Bogoni, J. A., Graipel, M. E., Castilho, P. V., Fantacini, F. M., Kuhnen, V. V., Luiz, M. R, Maccarini, T. B., Marcon, C. B., Teixeira, C. S. P., Tortato, M. A., Vaz-de-Mello, F. Z. and Hérnandez, M. I. M. (2016). Contributions of the mammal community, habitat structure, and spatial distance to dung beetle community structure. 
Biodiversity and conservation, v. 25, n. 9, p. 1661-1675.

Costa, F. C., Pessoa, K. K., Liberal, C. N., Filgueiras, B. K.; Salomão, R. P. and Iannuzzi, L. (2013) What Is the Importance of Open Habitat in a Predominantly Closed Forest Area to the Dung Beetle (Coleoptera, Scarabaeinae) Assemblage? Revista Brasileira de Entomologia, v. 57, n. 3, p. 329-334.

Cortes, T. P. B. B., Maciel, R. S., Nunes, M. F. H. and Souza, C. H. M. (2015) A infografia multimídia como recurso facilitador no ensino-aprendizagem em sala de aula. InterSciencePlace, v. 1, n. 29.

Favila, M.E. and Halffter, G. (1997) The use of indicator groups for measuring biodiversity as related to community structure and function. Acta Zool. Mexicana.,v. 72 , p. $1-25$.

Halffter, G. and Favila, M.E. (1993) The Scarabaeinae (Insecta: Coleoptera) an animal group for analyzing, inventorying and monitoring biodiversity in tropical rainforest and modified landscapes. Biol. Int. v. 27, p. 15-21.

Halffter, G. and Matthews, E. G. (1966) The natural history of dung beetles of the Subfamily Scarabaeinae (Coleoptera: Scarabaeidae). Folia Entomológica Mex., v. 45, p. $1-312$.

Hanski, I. and Cambefort, Y. (1991) Dung Beetle Ecology. Princeton, NJ: Princeton University Press, p. 51-67.

Hattwig, D., Bussert, K., Medaille, A. and Burgess, J. (2013). Visual Literacy Standards in Higher Education: New Opportunities for Libraries and Student Learning. Portal: Libraries and the Academy, v.13, n.1, p. 61-89.

Johri, P. and Misra, A. (2017). Digital technology in classroom: Changing the face of education infographic. In 2017 International Conference On Smart Technologies For Smart Nation (SmartTechCon), p. 405-406.

Kibar, P. N. and Akkoyunlu, B. (2014). A new approach to equip students with visual literacy skills: Use of infographics in education. In European Conference on Information Literacy, p. 456-465.

Lapolli, M., Vanzin, T. and Ulbricht, V. R. (2014). Infografia na web para a inclusão digital de alunos surdos. RENOTE, v.12, n.1.

Louzada, J. N. C. (2008) Scarabaeinae (Coleoptera: Scarabaeidae) detritívoros em ecossistemas tropicais: biodiversidade e serviços ambientais. Biodiversidade do solo em ecossistemas brasileiros, Lavras, Editora UFLA, p. 309-332.

Lundy, A. D. and Stephens, A. E. (2015). Beyond the literal: Teaching visual literacy in the 21 st century classroom. Procedia-Social and Behavioral Sciences, v.174, p. $1057-1060$.

Lyra, K. T., Oliveira, B. R. N., Reis, R. C. D. Cruz, W. M., Nakagawa, E. Y. and Isotani, S. (2016). Infográficos versus materiais de aprendizagem tradicionais: Uma investigação empírica. RENOTE, v..14, n.2, p.1-12.

Martix, S. and Hodson, J. (2014). Teaching with infographics: Practising new digital competencies and visual literacies. Journal of Pedagogic Development, v. 4, n.2, p. $17-27$.

Niebaum, K., Cunningham-Sabo, L., Carroll, J. and Bellows, L. (2015). Infographics: An Innovative Tool to Capture Consumers" Attention. Journal of Extension, v. 53, n.6, p.1-6.

Taguchi, K. and Ackerman, L. (2014) The Infographic: Is there a Place in Higher 
Education?. In: E-Learn: World Conference on E-Learning in Corporate, Government, Healthcare, and Higher Education. Association for the Advancement of Computing in Education (AACE), p. 1901-1905.

Tarasov, S. and Génier, F. (2015) Innovative Bayesian and Parsimony Phylogeny of Dung Beetles (Coleoptera, Scarabaeidae, Scarabaeinae) Enhanced by OntologyBased Partitioning of Morphological Characters. PLOS ONE, v. 10, n. 3.

Vaz-de-Mello F.Z. (2019) Scarabaeinae in Catálogo Taxonômico da Fauna do Brasil. PNUD. Disponível em: <http://fauna.jbrj.gov.br/fauna/faunadobrasil/127498>. Acesso em: 30 Jun. 2019.

Vaz-de-Mello, F.Z., Louzada, J.N.C. and Schoereder, J. H. (1998) New data and comments on scarabaeidae (Coleoptera: Scarabaeoidea) associated with attini (Hymenoptera: Formicidae). The Coleopterists' Bulletin, p. 209-216.

Yildirim, S. (2016) Infographics for Educational Purposes: Their Structure, Properties and Reader Approaches. Turkish Online Journal of Educational Technology-TOJET, v. 15, n. 3 , p. $98-110$. 